\title{
Mitochondrial Neurogastrointestinal Encephalomyopathy and Its Pathophysiology
}

\author{
Teruyuki Kurihara \\ Key words: Mitochondrial neurogastrointestinal encephalomyopathy, gastrointestinal symptoms, external \\ ophthalmoplegia, peripheral neuropathy, thymidine phosphorylase, mitochondrial DNA abnor- \\ malities
}

(DOI: 10.2169/internalmedicine.45.0148)

Mitochondrial neurogastrointestinal encephalomyopathy (MNGIE) is a very rare mitochondrial disorder with autosomal recessive inheritance (1). In the world literature fewer than 75 cases have been reported (2) and in Japan there have been only 4 cases of this disorder (3). This disorder has been more frequently identified in Mediterranean, Hispanic, Iranian-Jewish, and Ashkenazi-Jewish populations (2). The clinical features are characterized by (1) gastrointestinal symptoms including abdominal pain, vomiting, diarrhea, constipation, episodic pseudo-obstruction, which eventually causes severe weight loss and hypoproteinemia, (2) external ophathalmoplegia, (3) peripheral neuropathy, (4) proximal muscle weakness associated with mitochondrial abnormalities, and (5) leukoencephalopathy (1-5).

See also p 443.

DOI: $10.2169 /$ internal medicine.45.1371

It has been reported that MNGIE is caused by mutations in the gene encoding thymidine phosphorylase (TP). Leukocyte TP activity has been measured in controls: $0.67 \pm 0.21$ $\mu \mathrm{mol} / \mathrm{hr} / \mathrm{mg}(\mathrm{n}=19)$. Nishino et al reported that the mean of the 16 MNGIE patients had very low leukocyte TP activity: $0.009 \pm 0.021 \mu \mathrm{mol} / \mathrm{hr} / \mathrm{mg}$ (mean \pm SD) (1). Loss of function of this enzyme causes plasma accumulations of thimidine and deoxyuridine, which leads to mitochondrial nucleotide pool imbalance and affects mitochondrial DNA replication, and eventually leads to mitochondrial dysfunction (6). In the case of partial loss of TP activity the onset of the disease is late and the clinical symptoms are milder (7). MNGIE pa- tients have homozygous or compound heterozygous mutations in the thymidine phosphorylase gene, and also mitochondrial DNA deletion. Thirty different mutations in the TP gene have been identified, and most of the mutations are missense mutations (2). Recently a novel 18-base pair duplication in exon 8 of the TP gene was reported in a Spanish MNGIE patient (2). Though the disorder is caused by loss of function of TP, the cause of hypofunction of this enzyme may be either due to TP gene mutation, or combination of TP gene mutation and mtDNA deletion.

Kumagai $\mathrm{Y}$ et al (3) reported a 36-year-old Japanese woman of this disorder with thymidine phosphorylase (TP) gene mutation and the enzyme activity was low. Although the mother of this case had the same homozygotic S471L gene mutation, her enzyme activity was normal. Kumagai et al (3) analyzed 145 healthy persons, and the homyzygotic S 471L gene mutation was noted in $2.76 \%$ and their enzyme activity was normal. Among the Japanese, the prevalence of adult chronic progressive external ophthalmoplegia (CPEO) is 0.169 per 100,000 . CPEO is a mitochondrial disorder with a deletion mutation, and the external ophthalmoplegia is one of the characteristic manifestations of MNGIE as well. From the above results, Kumagai et al (3) calculated the incidence of MNGIE, $0.169 / 100,000 \times 0.0276=$ 0.0046644 per 100,000 , which is very close to the real incidence of Japanese MNGIE, that is only 4 cases in Japan. Kumagai et al (3) postulated that a single nucleotide polymorphism of the TP gene is a risk factor for MNGIE and mtDNA deletion may be related to decreased TP activity.

\section{References}

1. Nishino I, Spinazzola A, Papadimitriou A, et al. Mitochondrial neurogastrointestinal encephalomyopathy: An autosomal recessive disorder due to thymidine phosphorylase mutations. Ann Neurol
47: 792-800, 2000.

2. Gamez J, Lara MC, Mearin F, et al. A novel thymidine phosphorylase mutation in a Spanish MNGIE patient. J Neurol Sci 228:

Department of Internal Medicine Toho University Ohashi Medical Center, Division of Neurology

Correspondence to Teruyuki Kurihara, Department of Internal Medicine Toho University Ohashi Medical Center, Division of Neurology, 2-17-6 Ohashi, Meguro, Tokyo 153-8515 
DOI: $10.2169 /$ internalmedicine.45.0148

35-39, 2005.

3. Kumagai Y, Sugiura Y, Sugeno H, et al. Thymidine phophorylase gene mutation is not a primary cause of mitochondrial neurogastrointestinal encephalopathy (MNGIE). Intern Med 45: 443-446, 2006.

4. Nishigaki Y, Marti R, Copeland WC, Hirano M. Site-specific somatic mitochondrial DNA point mutations in patients with thymidine phosphorylase deficiency. J Clin Invest 111: 1913-1921, 2003.

5. Said G, Lacroix C, Planté-Bordeneuve V, et al. Clinicopathologi- cal aspects of the neuropathy of neurogastrointestinal encephalomyopathy (MNGIE) in four patients including two with CharcotMarie-Tooth presentation. J Neurol 252: 655-662, 2005.

6. Marti R, Spinazzola A, Nishino I, et al. Mitochondrial neurogastrointestinal encephalomyopathy and thymidine metabolism: results and hypotheses. Mitochondrion 2: 143-147, 2002.

7. Marti R, Verschuuren JJ, Buchman A, et al. Late-onset MNGIE due to partial loss of thymidine phosphorylase activity. Ann Neurol 58: 649-652, 2005 .

\section{(C) 2006 The Japanese Society of Internal Medicine http://www.naika.or.jp/imindex.html}

